\title{
THE DEVELOPMENT OF A GENERAL AUXILIARY DIAGNOSIS SYSTEM FOR COMMON DISEASE OF ANIMAL
}

\author{
Jianhua Xiao, Hongbin Wang*, Ru Zhang, Peixian Luan, Lin Li, Danning \\ $\mathrm{Xu}$ \\ College of Veterinary Medicine, NorthEast Agricultural University, Harbin,Heilongjiang \\ Province, P. R. China, 150030 \\ * Corresponding author, Address: College of Veterinary Medicine, NorthEast Agricultural \\ University, Harbin, 150030, Heilongjiang Province, P. R. China, Tel: +86-451-55191940, \\ Fax: +86-451-55190470, Email: neau1940@yahoo.com.cn
}

Abstract: In order to development one expert system for animal disease in china, and this expert system can help veterinary surgeon diagnose all kinds of disease of animal. The design of an intelligent medical system for diagnosis of animal diseases is presented in this paper. The system comprises three major parts: a disease case management system (DCMS), a Knowledge management system (KMS) and an Expert System (ES). The DCMS is used to manipulate patient data include all kinds of data about the animal and the symptom, diagnosis result etc. The KMS is used to acquire knowledge from disease cases and manipulate knowledge by human. The ES is used to perform diagnosis. The program is designed in N-layers system; they are data layer, security layer, business layer, appearance layer, and user interface. When diagnosis, user can select some symptoms in system group by system. One conclusion with three possibilities (final diagnosis result, suspect diagnosis result, and no diagnosis result) is output. By diagnosis some times, one most possible result can be get. By application, this system can increased the accurate of diagnosis to some extent, but the statistics result was not compute now.

Key words: expert system, diagnosis,

Please use the following format when citing this chapter:

Xiao, J., Wang, H., Zhang, R., Luan, P., Li, L. and Xu, D., 2009, in IFIP International Federation for Information Processing, Volume 294, Computer and Computing Technologies in Agriculture II, Volume 2, eds. D. Li, Z. Chunjiang, (Boston: Springer), pp. 953-958. 


\section{INTRODUCTION}

Computer-based methods are increasingly used to improve the efficient and effects of veterinary medical services. Those methods include both conventional techniques, such as database management systems(DBMSs), and Expert system (ES) techniques. From born of techniques, medical diagnosis and management has been a very active field(Silvia Alayon, 2007; Sengur, A, 2007; Xuewei Wang, 2004; I. Hatzilygeroudis, 1994). In medical diagnosis, database management system are used for storing, retrieving and generally manipulating patient data, whereas expert system are mainly used for performing diagnoses based on symptom of patient, since they can naturally represent the way experts reason. The structure of the paper is as follows.

\section{SYSTEM DESCRIPTION}

\subsection{The structure of system}

To develop one expert system for disease diagnosis, two matters needs to be done: collection of knowledge by typical symptom and the converting of experience into knowledge. There is only one difficult to be overcome. That is the latter of two works. The experiences were obtained from many cases by common symptoms. This experience can be converted into computer easily. But its adaptivity is limited by a lot of reasons. The experience obtained form one region may not applicable to another region. The experience obtained at past may not applicable to now. The disease is different in different region and time. Therefore it is very difficult to develop one ideal expert system. One general expert system for common disease of animal based on typical knowledge and common knowledge origin from cases was design and partly developed in this study. The structure of this expert system is show in figure.1.

It consists of three major parts: a disease case management system (DCMS), a Knowledge management system (KMS) and an Expert System (ES). The DCMS is used to manipulate patient data include all kinds of data about the animal and the symptom, diagnosis result, prescriptions, treatment results of disease case. After final diagnoses have been made, the patient animal cases are stored in the disease cases database. The KMS is used to acquire knowledge from disease cases and manipulate knowledge by human. The ES is used to perform diagnosis. The Knowledge Base (KB) contains the heuristic knowledge based on common symptom and knowledge based 
on typical symptom for diagnosing diseases. An augmented knowledge representation (KR) formalism based on production rules(see figure 2), the most widely employed KR formalism by ESs [3], is used. The Working Database (WDB) contains the case specific data, that is temp patient data, partial conclusions, data given by the user, and any other information relevant to the case under consideration. Patient data are transferred from the PDB. The Inference Engine (IE) uses the available knowledge to draw conclusions and make diagnoses. The Knowledge acquisition facility (KAF) is used for rule training. Finally, the UI performs a number of functions. The user can manipulate patient data and the cases. Also, the user can interact with the IE to start a diagnosis process as well as use the KAF to perform rule training.

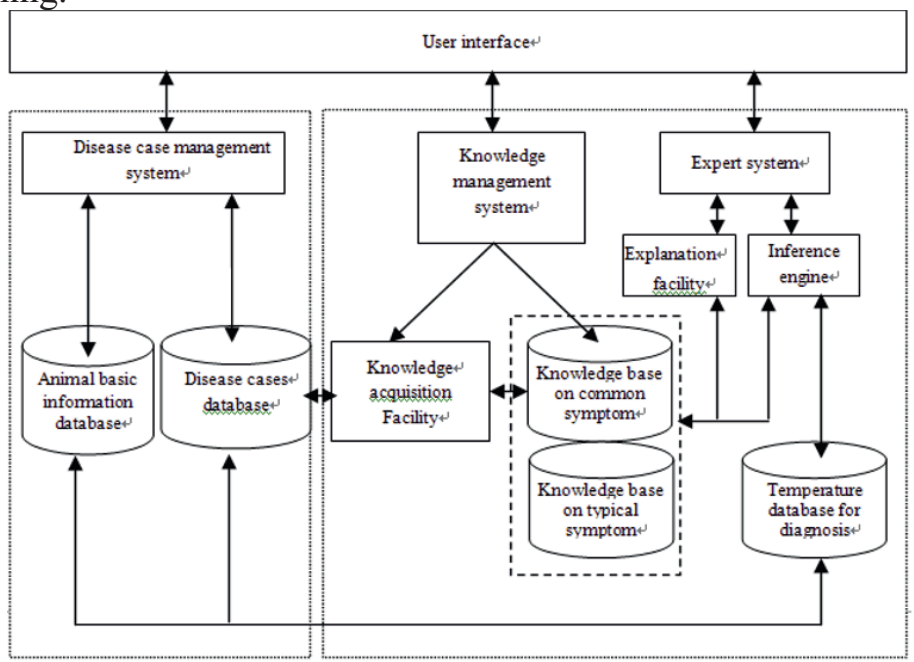

Fig. 1: The structure of animal disease diagnosis expert system

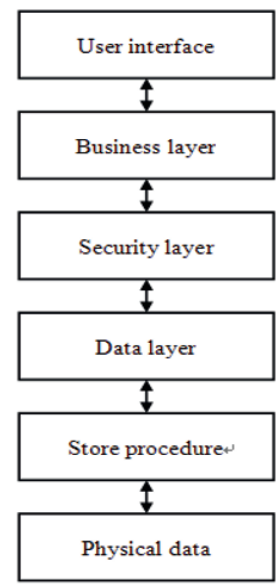

Fig. 2: The framework of cow disease diagnosis expert system 


\subsection{The system structure}

N-layers system was used in this program, they are data layer, security layer, business layer, appearance layer, and user interface.Data layer is one levis component that return data set to up-layer by communication with store procedure directly. Security layer take responsibility to validation role of user, password, user id. Business layer is one buffer area, it can protect the resources requested from client, and increasing flexibility significantly. Compare with traditionary three-layer structure, this structure has high security, high efficiency, and significant flexibility (see figure.3).

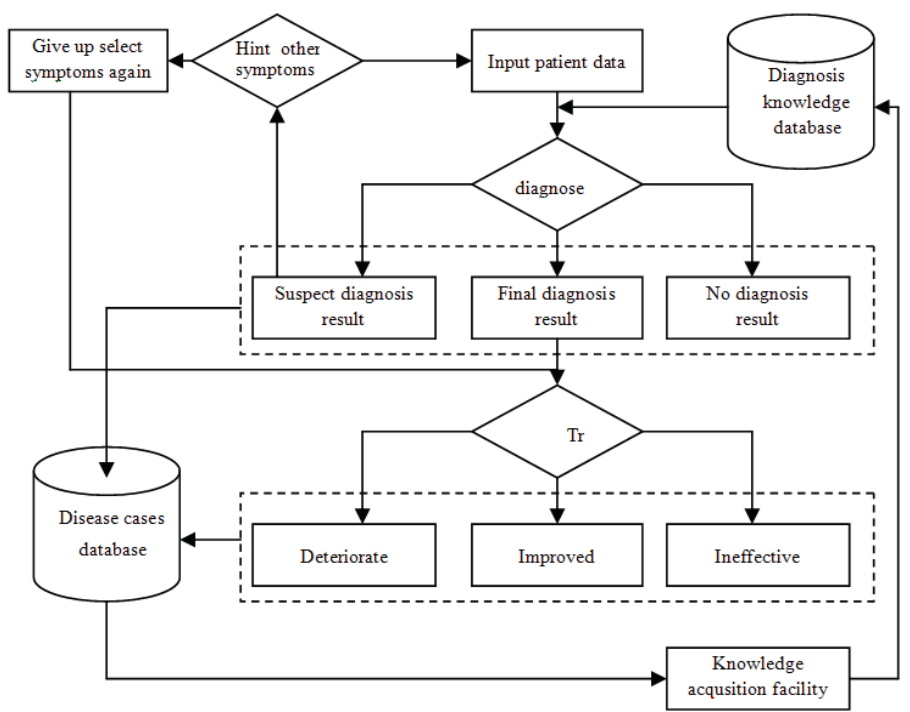

Fig. 3: the flow-sheet of operation for expert system

\section{3 flow-sheet of application}

Based on the symptom the user can select corresponding symptom in system group by system. The system will run inference procedure and make diagnosis by knowledge stored in database. One conclusion with three possibilities (final diagnosis result, suspect diagnosis result, and no diagnosis result) is output. If the result is suspect then the system will return user with a question: if the animal have symptom as these:", the system will list the possible symptoms by suspect disease in computer. The user can select symptom again. And diagnose further. Or treat as has got final diagnosis result. If there were no diagnosis result, then the system stops. If the treat procedure is run, some prescriptions will be provided and user can treat the animal with these prescriptions. These methods are adequate generally. The 
diagnosis result and treatment result will all be stored in disease cases database finally, the user must input the evaluation information for this diagnosis and treatment so as to train the knowledge in database. The knowledge acquisition facility will excavate knowledge from disease cases database.

\section{CONCLUSION}

The differential diagnosis can be complete from one class of disease in medical of human by doctor special in one subject. Under this condition, the degree of complexity will decreased greatly. Diagnosis will not be transfer to another doctor with different specialty until a well-pleasing result be produced. However, it is not so lucky that the doctor must give the ultimate answer of disease but not transfer to others because there were no one doctor that faced to one subject technically in veterinary more often. Any veterinary doctor must prescribe medicines whatever the disease belong to. There are more than 150 kinds of animal disease occurred usually. These diseases belong to 8 systems. Every disease will show several symptoms. Therefore, it is a more complex procedure of diagnosis for animal disease than disease of human being. Generally, common diseases of animal can be divided into infectious and noninfectious, noninfectious disease can be further divided into nervous system disease, digestive system, respiratory system, urinary system, circulation system, urinary system etc. diseases belong to one system have same symptoms, moreover these symptoms belong to one system generally. For example, respiratory system diseases have same symptoms such as cough, breathe heavily, respiratory sound etc. these symptoms belong to respiratory either. However, infectious diseases have more complex symptoms than infectious disease. Many symptoms belong to different system will show in one infectious diseases. Therefore, it is so difficult to make one decision of which one disease the animal can contract that any doctor is cautious without reliable experiment data or long year's experience. The diagnosis of disease is a very complex procedure especially in veterinary. The accurate of diagnosis depend on the knowledge of physician to some extent. But there was a great of difference between medicine and veterinary medicine. One physician may have plenty of experience in his profession because he will diagnose a great deal of case belong to his domain. He can study deeply in his profession either. But one veterinary can only meet a few cases in one domain and cases in every domain. Otherwise, the case can not be diagnosed to a very accurate level for short of precise and advanced equipment. The accurate was relative low in veterinary medical. There was more problem not solved in veterinary 
medical than human medical. Therefore one human doctor will get much knowledge than one veterinary doctor with same time and energy. Maybe the veterinary medicine has developed to the same level as human medicine in some area. But the level was relative low in most area. Based on above reason, a general expert system is more suitable than a lot of expert system special for one domain in veterinary practice.

\section{ACKNOWLEDGEMENTS}

Thanks to surgical department faculty working office of the Northeast Agricultural universdity, as well as Professor Liu Yun, associate professor Gao Li by the help regarding to this research.

\section{REFERENCES}

I. Hatzilygeroudis, P. J. Vassilakos, A. Tsakalidis. An Intelligent Medical System for Diagnosis of Bone Diseases, Proceedings of the 1st International Conference on Medical Physics and Biomedical Engineering (MPBE’94), Nicosia, Cyprus, May 1994, Vol. I, 148 152.

Sengur, A, \& Turkoglu, I., A hybrid method based on artificial immune system and fuzzy kNN algorithm for diagnosis of heart valve diseases , Expert Systems with Applications (2007), doi:10.1016/j.eswa.2007.08.003

Silvia Alayon, Richard Robertson, Simon K. Warfield, et al. A fuzzy system for helping medical diagnosis of malformations of cortical development. Journal of Biomedical Informatics, 40 (2007) $221-235$

Xuewei Wang, Haibin Qu, Ping Liu, Yiyu Cheng. A self-learning expert system for diagnosis in traditional Chinese medicine, Expert Systems with Applications 26 (2004) 557-566 